\title{
A narrative review of progress in diagnosis and treatment of small cell lung cancer patients with hyponatremia
}

\author{
Ranpu Wu ${ }^{1}$, Chuling $\mathrm{Li}^{2}$, Zimu Wang ${ }^{3}$, Hang Fan ${ }^{2}$, Yong Song ${ }^{1,2}$, Hongbing Liu ${ }^{1,2}$ \\ ${ }^{1}$ Department of Respiratory and Critical Care Medicine, Jinling Hospital, Southeast University of Medicine, Nanjing, China; ${ }^{2}$ Department of \\ Respiratory and Critical Care Medicine, Affiliated Jinling Hospital, Medical School of Nanjing Medical University, Nanjing, China; ${ }^{3}$ Department of \\ Respiratory Medicine, Jinling Hospital, Nanjing University School of Medicine, Nanjing, China \\ Contributions: (I) Conception and design: R Wu; (II) Administrative support: None; (III) Provision of study materials or patients: None; (IV) \\ Collection and assembly of data: None; (V) Data analysis and interpretation: None; (VI) Manuscript writing: All authors; (VII) Final approval of \\ manuscript: All authors. \\ Correspondence to: Hongbing Liu, PhD; Yong Song, PhD. Department of Respiratory and Critical Care Medicine, Affiliated Jinling Hospital, Medical \\ School of Nanjing University, 305 East Zhongshan Road, Nanjing 210002, China. Email: netlhb@126.com; yong_song6310@yahoo.com.
}

\begin{abstract}
Small cell lung cancer (SCLC) is one of the malignant cancers of lung tumors, and hyponatremia, defined as serum sodium concentration $\left(\mathrm{Na}^{+}\right)$lower than $135 \mathrm{mmol} / \mathrm{L}$, is the most common complication of solid tumors, with an incidence of up to $18.9 \%$ and a negative impact on quality of life in SCLC. As a prognostic index of SCLC, timely monitoring and correcting of hyponatremia is of great clinical significance for prolonging the survival period of patients. In the explore of new drugs for small cell lung cancer, it is necessary to include hyponatremia as an evaluation index in clinical studies. As the occurrence of hyponatremia is sometimes unavoidable owing to SCLC specific neurological characteristics, early monitoring to detect the presence of hyponatremia and timely correction are helpful to improve the prognosis of patients. There are many predisposing factors for hyponatremia, including heterotopia of antidiuretic hormone (ADH), use of platinum-based chemotherapy drugs, and intracranial metastasis, among others. Patients with small cell lung cancer are usually asymptomatic in the early stage, while it is of great significance to find a suitable clinical index to judge whether it is a malignant inducement or not. In the clinical setting, due to different electrolyte levels and therapeutic scheduling for the primary disease, an individualized plan is often made, mainly comprising water restriction, infusion, and medications. This review includes related clinical studies and describes the common symptoms and predisposing factors of hyponatremia in patients with SCLC, and their impact on quality of life and prognosis.
\end{abstract}

Keywords: Small cell lung cancer (SCLC); hyponatremia; syndrome of inappropriate antidiuretic hormone secretion (SIADH); tolvaptan

Submitted Sep 24, 2020. Accepted for publication Nov 15, 2020.

doi: $10.21037 /$ tlcr-20-1147

View this article at: http://dx.doi.org/10.21037/tlcr-20-1147

\section{Introduction}

Small cell lung cancer (SCLC) is a malignant tumor with strongly unfavorable outcomes, thus early diagnosis and treatment of the disease is key to the survival and prognosis of those afflicted $(1,2)$. Many patients have no choice but to undergo chemotherapy combined with radiotherapy swiftly following diagnosis (3). Hyponatremia, as a serious complication of lung cancer, occurs in $25-47 \%$ of patients (4); while the incidence appears high, $36 \%$ of them predominantly manifests mildly when correlated with lung cancer (5). Compared with other tumors, severe hyponatremia tends to take place particularly in SCLC, accounting for $19.9 \%$ (6). Unfortunately, hyponatremia encroaches on the quality of life of SCLC patients.

We present the following article in accordance with the 
Narrative Review reporting checklist (available at http:// dx.doi.org/10.21037/tlcr-20-1147).

\section{Development of hyponatremia}

The etiology of hyponatremia is complex, and there are many reasons why patients develop hyponatremia. Hyponatremia may arise in correlation with: (I) stroke and other primary mental primary diseases (7); (II) carbamazepine (8), cyclophosphamide, and selective serotonin reuptake inhibitors (SSRI) (9), exogenous hormones and other drugs; (III) primary tumors (10); (IV) hypophysis disease, hypothyroidism and adrenal insufficiency (11); (V) surgery; (VI) lung diseases such as pneumonia; (VII) hereditary syndrome of inappropriate antidiuretic hormone secretion (SIADH) (12). Hyponatremia often occurs insidiously, with complicated etiology.

The most serious and malignant inducement for hyponatremia is primary malignant tumors, especially SCLC. In the case of SCLC, after excluding other etiologies, the risk of hyponatremia is increased by the therapeutic schedule for the primary disease, SIADH caused by paraneoplastic syndrome, and also disease progression to the brain.

\section{Primary lung cancer treatment}

In the treatment of primary lung cancer, different platinum groups have varying side effects due to divergent characteristics of nephrotoxicity. The incidence of hyponatremia was higher in patients treated with cisplatin than carboplatin $(13.5 \%$ vs. $7.6 \% ; \mathrm{P}<0.01)(6)$. Cisplatin, a traditional and effective drug for lung cancer, has shown greater gastrointestinal and renal toxicity (13), which are both considered causes of hyponatremia (14). In a study of 281 patients, 12 (4.3\%) cases of short-term decrease in serum sodium after using cisplatin were recorded (15).

At the same time, in a retrospective study with 46 advanced non (N)SCLC patients, it was speculated that pemetrexed itself may lead to sodium imbalance, which was unrelated to the combined administration of platinum therapy (16). Although the incidence of hyponatremia observed during pemetrexed maintenance therapy was very high, there was an obvious limitation in this study; the correlation between pemetrexed and hyponatremia was discovered accidentally, so further prospective clinical trials are required to specifically evaluate the role of pemetrexed in hyponatremia.
Recently, immunotherapies have also been reported to be at risk of aggravating hyponatremia in lung cancer (17). Nivolumab is one such targeted therapy, which induces adrenal insufficiency and causes hyponatremia (18).

\section{Syndrome of inappropriate secretion of antidiuretic hormone (ADH)}

The incidence of SIADH in patients with SCLC is $10-16 \%$, yet only $2-4 \%$ in NSCLC patients (19-21). Bartter and Schwartz formulated the diagnostic standard for SIADH in 1967 (22), and it is still in use today. Distinguishing SIADH from other causes of hyponatremia, particularly salt wasting syndrome and other hypovolemic states, is significant for selecting the appropriate treatment and ensuring that serum sodium abnormalities do not deteriorate. Caused by ectopic and unregulated secretion of ADH, SIADH patients exhibit combined water retention and secondary solute loss. The overall solute loss in patients with chronic SIADH is more prominent than water retention (23).

Some scholars believe that SIADH may result from the ectopic production of arginine vasopressin (AVP) by tumor cells, or the enhancement of AVP effects by anticancer drugs or palliative drugs (24). However, it has been found that a third of lung cancer patients with hyponatremia have no evidence of ectopic production of AVP (25), but instead display an increase in cardionatrin (26).

\section{Disease progression like brain metastasis}

Similarly, brain metastases may lead to excessive sodium loss due to salt wasting syndrome, yet this is usually difficult to distinguish from SIADH $(27,28)$. However, distinction is necessary, as choosing the wrong treatment method will lead to deterioration of hyponatremia.

\section{Clinical symptoms}

Generally, hyponatremia cannot be detected in the early stage in many patients, and appears merely as a cluster of non-specific symptoms (29). Acute sodium imbalance (125$130 \mathrm{mEq} / \mathrm{L}$ ) leads to nausea and discomfort due to the rapid decrease of blood sodium, and leads to vomiting, headache, drowsiness, and other neurological symptoms (30). If the serum sodium level drops to $115-120 \mathrm{mEq} / \mathrm{L}$, coma and respiratory arrest may occur, endangering the patient's life. Although the symptoms caused by acute hyponatremia are usually reversible, they can also cause permanent 
Table 1 Relationship between hyponatremia and prognosis

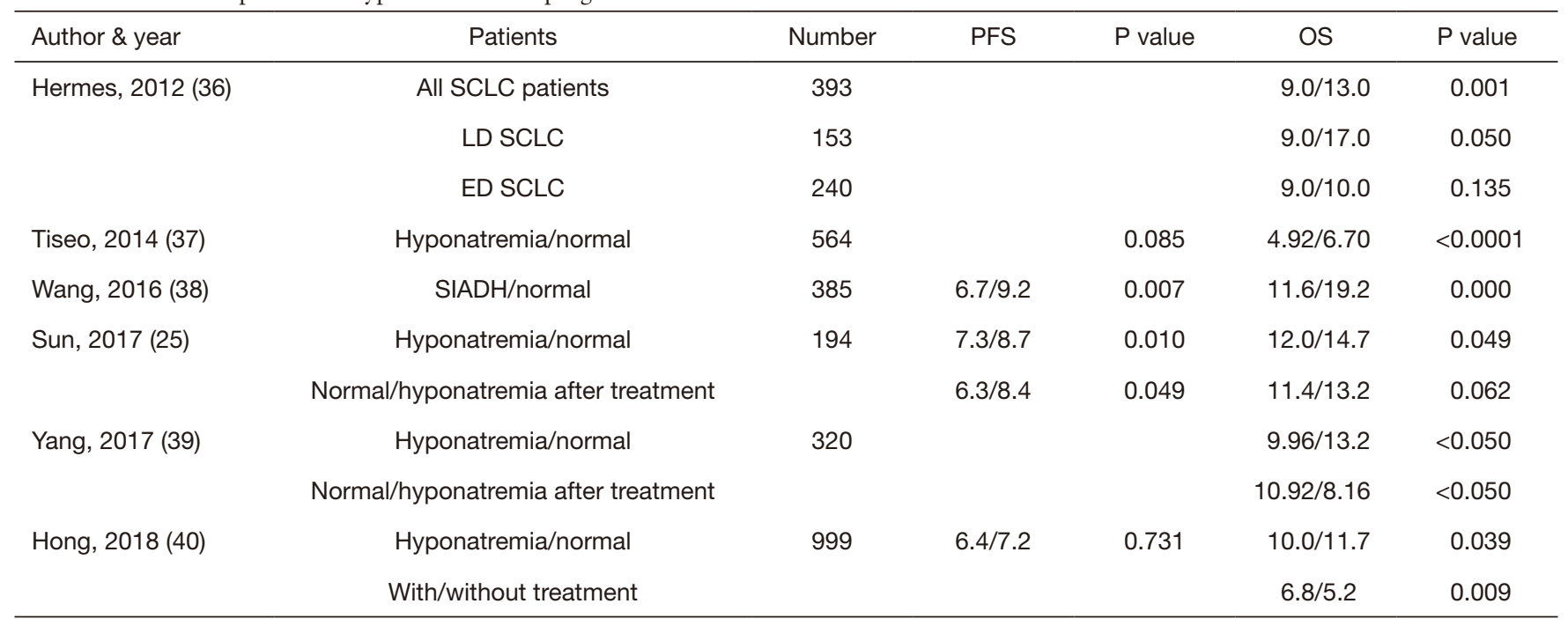

SCLC, small cell lung cancer; LD, limited disease; ED, extensive disease; OS, overall survival; PFS, progression free survival.

neurological damage.

Due to the unique adaptive mechanism of the brain, patients with chronic hyponatremia remain asymptomatic, even when the serum sodium concentration is $<120 \mathrm{mmol} / \mathrm{L}$. However, if patients have mild to moderate hyponatremia for an extended time, falls and gait problems (31), and also fractures may happen, especially in patients $>65$ years old (32).

\section{Hyponatremia can be a prognostic factor in SCLC}

As a highly malignant tumor, the standard treatment for SCLC is chemotherapy combined with radiotherapy, yet results are often unsatisfactory, leaving patients with a poor quality of life, and serious side effects from the chemotherapy regimens $(33,34)$. Hyponatremia could be a prognostic factor in patients with SCLC, similar to NSCLC (35), as it shows a serious impact on disease progression and quality of life in patients with SCLC. Several studies explored statistical significance of hyponatremia in small cell lung cancer, listed in Table 1.

Sengupta et al. performed a cross-sectional study including 116 patients with lung cancer (2), among whom hyponatremia could be seen in $44.8 \%$. There was a negative correlation between the ECOG score and serum sodium status measured at admission. The results of 105 cancer patients indicated a statistical relationship between increased hospitalization time and hyponatremia (41). Hospital mortality and cost were especially increased among patients with severe hyponatremia.

The results of Cox multivariate analysis have shown hyponatremia to be associated with worse prognosis $[\mathrm{P}=0.0024$, hazard ratio $(\mathrm{HR})=1.44,95 \%$ confidence interval (CI): 1.13-1.82) and lower median survival $(\mathrm{P}<0.0001, \mathrm{HR}=1.67,95 \% \mathrm{CI}: 1.32-2.10)(37)$. This retrospective study demonstrated that hyponatremia was an independent prognostic factor for topotecan treatment, but prospective studies are needed to confirm this result (37).

A study (42) by Abu Zeinah et al. also concluded that mortality in patients with moderate to severe hyponatremia was 4.28 times higher than that in normal people $(\mathrm{P}<0.05)$, which supported that hyponatremia can be used as a risk factor for hospital death. At the same time, in a retrospective study, normalization of sodium concentration was shown to help prolong the total survival time and progressionfree survival (PFS). Hansen's study (43) suggested that there was a statistical difference in the effect of normalization of sodium hemorrhage on the outcome of SCLC patients. These studies have shown that hyponatremia may be an independent predictor of prognosis in SCLC patients, not just for NSCLC (44).

Another retrospective study analyzed 999 SCLC patients, of whom 226 patients with hyponatremia had shorter overall survival (OS) than those with normal sodium levels (11.7 vs. 10.0, $\mathrm{P}=0.039)$, and 119 patients with untreated hyponatremia also showed shorter OS (6.8 vs. 5.2, $\mathrm{P}=0.009$ ) (40). However, hyponatremia did not show significance in assessing the factors influencing PFS (7.2 
vs. $6.4, \mathrm{P}=0.731$ ). In yet another retrospective study of 395 patients with SCLC, hyponatremia was also associated with significantly shorter median survival (9.0 vs. 13.0, $\mathrm{P}<0.001$ ) (36).

The work of Castillo et al. showed the death rate of SCLC patients to be the highest (86\%), and the data does show a correlation between hyponatremia and PFS, but no significant difference was found, which may be caused by the high degree of malignancy and rapid progression of SCLC itself. Even if hyponatremia had a trend instead of no significant effect on PFS in this study, it could still statistically be an independent predictor for OS in SCLC patients to evaluate patient outcomes, which require early detection and clinical correction (45).

Correcting hyponatremia can ameliorate prognosis to an extent (39). Studies have found that the mean survival time of patients with corrected hyponatremia in the hyponatremia group was significantly longer than the patients with uncorrected hyponatremia $(t=2.75, \mathrm{P}<0.05)(46)$.

\section{Treatment}

Early detection and correction are of great significance to improve quality of life and reduce the risk of death (47). When making a treatment plan, clinicians should consider blood volume, electrolyte level, complications of neurological symptoms (48), and social and psychological factors, including the wishes of patients. Also, $\mathrm{Na}^{+}$ concentration should be continuously monitored during subsequent treatment.

\section{Fluid restriction and salt supplementation}

For SCLC patients with mild to moderate symptoms, fluid restriction is feasible, with a target of $<800 \mathrm{~mL}$ per day. Unfortunately, response to this approach is slow, and its ability to increase serum sodium is weak. For severe hyponatremia, hypertonic saline is the first choice; when the blood volume is normal, isotonic saline may reduce the serum sodium level and aggravate hyponatremia. However, even if it leads to a rapid increase in the level of serum sodium, it can only be used as an initial treatment, as it is difficult to maintain serum sodium content for an extended period.

If hyponatremia persists, sodium chloride may be given orally in the form of salt tablets or intravenously in the form of saline. Diuretics, such as furosemide, can also be added to salt tablets $20 \mathrm{mg}$ twice a day to dilute urine concentration and increase water excretion.
Studies have explored the differences between treatment options, like fluid restriction with or without furosemide and $\mathrm{NaCl}$. However, in patients with SIADH, there has been no statistical difference in changes of sodium levels (49).

Case reports have indicated some SCLC patients had good tolerance and response to therapy of limited infusion combined with salt intake, when undergoing chemotherapy with cisplatin and etoposide (50).

At the same time, patients with increased cardionatrin levels had no response to fluid restriction; therefore, if the sodium intake cannot be increased concurrently, liquid restriction may exacerbate hyponatremia in those patients (51).

\section{First aid for nervous symptoms}

The diagnosis of hyponatremia requires laboratory tests, but in severe and symptomatic cases, first aid is often required before laboratory results are available to identify the cause $(52,53)$.

Notably, the presence of neurological symptoms at admission determine if undergoing emergency treatment with hypertonic saline is required (54). If symptoms such as seizures and delirium occur, hypertonic saline infusion should be performed immediately. Large doses of $3 \%$ hypertonic saline need to be administered within the first 3-4 hours, and sodium levels should be monitored within 2-3 hours to adjust the dose. A rise of 3-4 mEq/L in the first few hours of treatment is acceptable. If the patient's mental status does not improve, another $100 \mathrm{~mL}$ of hypertonic saline can be injected until symptoms are relieved.

\section{Vasopressin $\mathbf{V} 2$ receptor antagonists like tolvaptan}

In chemotherapy treatment of SCLC patients, the method of fluid restriction has limitations and often requires the use of drug therapy. As undergoing chemotherapy requires a massive infusion, the blood sodium level of many patients cannot be controlled with basic water and dietary restrictions. Vasopressin V2 receptor antagonists like conivaptan, tolvaptan, and mozavaptan have been introduced into clinical practice, and among them, tolvaptan has been widely used for this purpose.

For a 45-year-old patient, hyponatremia became especially difficult to control with standard therapy after several cycles of chemotherapy, but was finally successfully controlled by oral administration of tolvaptan. The 
administration of tolvaptan was stopped after tumor metastasis occurred, this led to the recrudescence of hyponatremia, and the patient died about 1 year after diagnosis (55). An 88-year-old patient underwent a similar course of treatment, refractory hyponatremia was controlled by tolvaptan, and finally relapsed after discontinuation.

For SCLC patients with a diagnosis of SIADH, vasopressin receptor antagonists like tolvaptan have shown clinical benefit (56). However, tolvaptan is hepatotoxic and should not be given to patients with liver disease (57).

A prospective study including 10 SCLC patients with severe SIADH concluded that patients can benefit from tolvaptan, which also eliminates risk of long-term hospitalization, and improves quality of life (21). As tolvaptan can be used in outpatients, it can eliminate anxiety related to dietary restrictions and improve enthusiasm towards treatment.

A meta-analysis of 520 patients showed that tolvaptan could reduce adverse events compared with other antagonists, but could not significantly reduce mortality (58). Berardi et al.'s study included 90 cancer patients, 37 (41\%) were treated with tolvaptan and labeled group A, while the remaining patients are treated with hypertonic and saline solutions, diuretics, and fluid restriction, and were labeled group B. Except a few cases of thirst (16\%), no significant toxicity caused by tolvaptan was observed among the participants (22). Other uncommon side effects of tolvaptan include hypoglycemia, hyperuricemia, syncope, dizziness, headache, general discomfort, and diarrhea.

Low-dose tolvaptan has been found to be more effective than a high-salt diet and fluid restriction in controlling hyponatremia, with fewer complications such as hypernatremia and liver dysfunction. Patients who did not receive tolvaptan had significantly longer hospital stays, and many did not reach sodium-adjusted values during hospitalization (22).

\section{Other therapies used in clinic}

Intravenous tolvaptan is very effective in correcting hyponatremia and mental status in hospitalized patients. Other drugs, including urea, demeclocycline, and fludrocortisone are also used clinically (59).

However, the taste of urea is unacceptable to many patients, and it does not correct serum sodium levels quickly, so its use is very limited (60). Demeclocycline, a tetracycline analogue, can eliminate the effects of $\mathrm{ADH}$ at the renal tubular level, but its effectiveness may be delayed, taking up to 1-2 weeks.

Similarly, demeclocycline causes reversible azotemia and nephrotoxicity, especially in patients with renal insufficiency, whereas demeclocycline is at risk of inducing renal diabetes insipidus. Lithium is also effective, but is nephrotoxic for SIADH patients. Therefore, mentioned therapies above are recommended only when others have failed.

Fludrocortisone has shown the function of correcting hyponatremia under monitoring of side effects like hypokalemia, body fluid overload, and hypertension. A report has shown that after using hypertonic saline to improve acute nervous system symptoms, $0.1 \mathrm{mg}$ of fludrocortisone twice a day can control hyponatremia successfully in SCLC patients with severe hyponatremia (61).

\section{Monitoring blood sodium to prevent complications}

Correcting serum sodium too quickly in chronic hyponatremia may lead to the occurrence of osmotic demyelination syndrome. Due to the existence of an adaptive mechanism which protects the brain from swelling, the serum sodium level should be raised in a controllable way; the correction rate should be maintained at $<12 \mathrm{mEq} / \mathrm{L}$ within 24 hours, and $<18 \mathrm{mEq} / \mathrm{L}$ within 48 hours. Meanwhile, some studies have advocated a correction rate of no more than $8 \mathrm{mEq} / \mathrm{L}$ per 24 hours, or no more than $0.5-1 \mathrm{mEq} / \mathrm{L}$ per hour to protect the brain from permeability demyelination (62).

In general, if serum sodium concentration is consistently below $136 \mathrm{mEq} / \mathrm{L}$ and the increase within first 24 hours is $<5 \mathrm{mEq} / \mathrm{L}$, the dose of tolvaptan should be increased. If serum sodium rises $>145 \mathrm{mEq} / \mathrm{L}$ or increases faster than $12 \mathrm{mEq} / \mathrm{L}$ within 24 hours of treatment or $>8 \mathrm{mEq} / \mathrm{L}$ within the first 8 hours of treatment, the dose should be reduced (63).

Studies have suggested that sodium should be monitored regularly upon commencement of tolvaptan to avoid overcorrection of sodium (64). Usually, it is recommended that serum sodium is measured every $4-6$ hours, and this frequency should be increased in patients who are at risk of osmotic demyelination. Once primary cancer progressed, SCLC patients need continuously monitor after adjusting new therapy in case hyponatremia occurs again.

\section{Discussion}

SCLC accounts for $15 \%$ of all lung cancer cases (65). A retrospective cohort analysis sampled 1,758 patients 
from 2 large, integrated delivery networks (IDN) (45); after excluding 456 hypovolemic hyponatremia (3\%) patients, this study included patients with SCLC, NSCLC, lymphoma, and so on. It was found that $75.8 \%$ of NSCLC patients and $76.3 \%$ of SCLC patients showed hyponatremia, which was much higher than the 20-50\% reported previously. Moderate or severe hyponatremia was found in $46 \%$ of SCLC patients, and $24.7 \%$ of elderly hospitalized patients suffered hyponatremia (66). The occurrence of hyponatremia is particularly prominent and seriously affects the quality of life (65).

Clinically, patients with SCLC are often asymptomatic in the early stage, and hyponatremia is often overlooked (67). Patients are usually admitted to hospital for hyponatremia detected by electrolyte examination, and SCLC is eventually diagnosed after relevant examinations are completed. Therefore, it is of great significance to find a suitable clinical index to identify whether it is a malignant inducement.

Many researchers are currently exploring whether markers such as D-dimer are related to malignant hyponatremia (68). Glycoprotein CD56, also known as NCAM1, is a member of the neural cell adhesion molecule family, and abnormal expression of CD56 can be seen in solid tumors, especially SCLC. Studies have speculated that CD56 could be used as a diagnostic biomarker to identify whether the tumor is of a neuroendocrine source (69).

Liquid biopsy is considered a promising technique for early diagnosis of SCLC, and encompasses markers like neuron-specific enolase (NSE) $(70,71)$. Studies have suggested that copeptin may be a diagnostic tool for differentiating malignant from non-malignant hyponatremia. In a prospective study, 146 patients with SIAD were included, 39 of whom were diagnosed with cancer-related hyponatremia (72). Although the final result failed to prove that copeptin can be a marker of malignant disease $(\mathrm{P}>0.05)$, the proportion of tumor etiology in patients with hyponatremia was still considerable. The risk of tumors, especially SCLC, should be considered in refractory hyponatremia after excluding other factors (73).

Because of the short PFS, high recurrence rate, and rapid progress of SCLC, many studies are devoted to exploring the subsequent drug selection for patients who failed firstline treatment. The UK Coordination Committee assessed 4,000 patients and concluded that hyponatremia was a significant prognostic factor for SCLC, and that it needs to be incorporated in the design of SCLC clinical trials and used as an evaluation index when assessing the efficacy of new drugs.
In on-going trials, hyponatremia has already been used as a prognostic marker of erlotinib in the treatment of NSCLC (74), and phase I and phase II clinical studies of irinotecan in lung cancer $(75,76)$. Antiangiogenic therapy, targeted therapy, and immunotherapy as new therapies are more commonly used in the treatment of SCLC, and many regimens have shown their merits in reducing side effects such as hyponatremia (77-79).

Unfortunately, regardless of the treatment regimen used, occurrence of hyponatremia is sometimes unavoidable in SCLC due to the neurological characteristics of the disease. For patients who have already developed hyponatremia, it is necessary to formulate an individual diagnosis and treatment plan according to the serum sodium concentration and clinical symptoms. Standard hyponatremia treatment can be formulated according to the status of blood volume, including fluid restriction, normal saline, or $3 \%$ hypertonic saline infusion. When these therapies fail to improve the clinical condition and patients need to start chemotherapy immediately, tolvaptan $15 \mathrm{mg} / \mathrm{d}$ can be used as the initial dose, with other hyponatremia treatments stopped 12 hours earlier. During tolvaptan treatment, no fluid restriction is required to ensure the patient chemotherapy needs.

Because fluid restriction worsens nutritional status, tolvaptan is also preferred in malnourished patients. The use of $\mathrm{ADH}$ receptor antagonists can improve hyponatremia symptoms even in patients with advanced SCLC who are unable to receive chemotherapy and must receive palliative treatment instead (76).

\section{Acknowledgments}

Funding: None.

\section{Footnote}

Reporting Checklist: The authors have completed the Narrative Review reporting checklist. Available at http:// dx.doi.org/10.21037/tlcr-20-1147

Conflicts of Interest: All authors have completed the ICMJE uniform disclosure form (available at http://dx.doi. org/10.21037/tlcr-20-1147). The authors have no conflicts of interest to declare.

Ethical Statement: The authors are accountable for all aspects of the work in ensuring that questions related to the accuracy or integrity of any part of the work are 
appropriately investigated and resolved.

Open Access Statement: This is an Open Access article distributed in accordance with the Creative Commons Attribution-NonCommercial-NoDerivs 4.0 International License (CC BY-NC-ND 4.0), which permits the noncommercial replication and distribution of the article with the strict proviso that no changes or edits are made and the original work is properly cited (including links to both the formal publication through the relevant DOI and the license). See: https://creativecommons.org/licenses/by-nc-nd/4.0/.

\section{References}

1. Higgins KA, Gorgens S, Sudmeier LJ, et al. Recent developments in limited stage small cell lung cancer. Transl Lung Cancer Res 2019;8:S147-52.

2. Sengupta A, Banerjee SN, Biswas NM, et al. The Incidence of Hyponatraemia and Its Effect on the ECOG Performance Status among Lung Cancer Patients. J Clin Diagn Res 2013;7:1678-82.

3. Nesbit EG, Leal TA, Kruser TJ. What is the role of radiotherapy for extensive-stage small cell lung cancer in the immunotherapy era? Transl Lung Cancer Res 2019;8:S153-62.

4. Zheng Y, Zheng FP, Li H. The prevalence and causes of hyponatremia in hospitalized patients. Zhonghua Nei Ke Za Zhi 2020;59:29-34.

5. Thajudeen B, Salahudeen AK. Role of tolvaptan in the management of hyponatremia in patients with lung and other cancers: current data and future perspectives. Cancer Manag Res 2016;8:105-14.

6. Ezoe Y, Mizusawa J, Katayama H, et al. An integrated analysis of hyponatremia in cancer patients receiving platinum-based or nonplatinum-based chemotherapy in clinical trials (JCOG1405-A). Oncotarget 2018;9:6595-606.

7. Liamis G, Barkas F, Megapanou E, et al. Hyponatremia in Acute Stroke Patients: Pathophysiology, Clinical Significance, and Management Options. Eur Neurol 2019;82:32-40.

8. Lu X, Wang X. Hyponatremia induced by antiepileptic drugs in patients with epilepsy. Expert Opin Drug Saf 2017; 16:77-87.

9. Liang Teo DC, Mok VWL. 127 Successful Treatment of Major Depressive Disorder with Moclobemide After Recurrent Hyponatremia Induced by Multiple Antidepressant Classes. CNS Spectr 2020;25:280-1.

10. Mackelaite L, Lederer E. Hyponatremia Complicating
Esophageal Carcinoma: A Challenging Differential Diagnosis. Am J Med Sci 2018;356:567-9.

11. Liamis G, Filippatos TD, Liontos A, et al. MANAGEMENT OF ENDOCRINE DISEASE: Hypothyroidism-associated hyponatremia: mechanisms, implications and treatment. Eur J Endocrinol 2017;176:R15-R20.

12. Yasir M, Mechanic OJ. Syndrome of Inappropriate Antidiuretic Hormone Secretion (SIADH). StatPearls. Treasure Island (FL): StatPearls Publishing Copyright (C) 2020, StatPearls Publishing LLC.; 2020.

13. Raftopoulos H. Diagnosis and management of hyponatremia in cancer patients. Support Care Cancer 2007;15:1341-7.

14. Liamis G, Filippatos TD, Elisaf MS. Electrolyte disorders associated with the use of anticancer drugs. Eur J Pharmacol 2016;777:78-87.

15. Iyer AV, Krasnow SH, Dufour DR, et al. Sodium-wasting nephropathy caused by cisplatin in a patient with small-cell lung cancer. Clin Lung Cancer 2003;5:187-9.

16. Gota V, Kavathiya K, Doshi K, et al. High plasma exposure to pemetrexed leads to severe hyponatremia in patients with advanced non small cell lung cancer receiving pemetrexed-platinum doublet chemotherapy. Cancer Manag Res 2014;6:261-5.

17. Cantini L, Merloni F, Rinaldi S, et al. Electrolyte disorders in advanced non-small cell lung cancer patients treated with immune check-point inhibitors: A systematic review and meta-analysis. Crit Rev Oncol Hematol 2020;151:102974.

18. Rai M, Go M. Nivolumab Induced Adrenal Insufficiency: Rare Side-effect of a New Anti-cancer Therapy - Immunecheckpoint Inhibitors. Cureus 2020;12:e7625.

19. Agarwal KA, Soe MH. Beyond the Dual Paraneoplastic Syndromes of Small-Cell Lung Cancer with ADH and ACTH Secretion: A Case Report with Literature Review and Future Implications. Case Rep Oncol Med 2018;2018:4038397.

20. Miyashita K, Matsuura S, Naoi H, et al. Successful treatment by tolvaptan of the syndrome of inappropriate antidiuretic hormone secretion that may be associated with chemotherapy-induced tumour lysis in a patient with small-cell lung carcinoma. Respirol Case Rep 2018;6:e00296.

21. Petereit C, Zaba O, Teber I, et al. A rapid and efficient way to manage hyponatremia in patients with SIADH and small cell lung cancer: treatment with tolvaptan. BMC Pulm Med 2013;13:55. 
22. Berardi R, Mastroianni C, Lo Russo G, et al. Syndrome of inappropriate anti-diuretic hormone secretion in cancer patients: results of the first multicenter Italian study. Ther Adv Med Oncol 2019;11:1758835919877725.

23. Kitchlu A, Rosner MH. Hyponatremia in patients with cancer. Curr Opin Nephrol Hypertens 2019;28:433-40.

24. Platania M, Verzoni E, Vitali M. Hyponatremia in cancer patients. Tumori 2015;101:246-8.

25. Sun NH, Wang SH, Liu JN, et al. The productions of atrial natriuretic peptide and arginine vasopressin in small cell lung cancer with brain metastases and their associations with hyponatremia. Eur Rev Med Pharmacol Sci 2017;21:4104-12.

26. Mok T, Ladrera G, Srimuninnimit V, et al. Tumor marker analyses from the phase III, placebo-controlled, FASTACT-2 study of intercalated erlotinib with gemcitabine/platinum in the first-line treatment of advanced non-small-cell lung cancer. Lung Cancer 2016;98:1-8.

27. Lannou A, Carrie C, Rubin S, et al. Salt wasting syndrome in brain trauma patients: a pathophysiologic approach using sodium balance and urinary biochemical analysis. BMC Neurol 2020;20:190.

28. Oh JY, Shin JI. Syndrome of inappropriate antidiuretic hormone secretion and cerebral/renal salt wasting syndrome: similarities and differences. Front Pediatr 2015;2:146.

29. Peri A. Management of hyponatremia: causes, clinical aspects, differential diagnosis and treatment. Expert Rev Endocrinol Metab 2019;14:13-21.

30. Fuca G, Mariani L, Lo Vullo S, et al. Weighing the prognostic role of hyponatremia in hospitalized patients with metastatic solid tumors: the HYPNOSIS study. Sci Rep 2019;9:12993.

31. Negri AL, Ayus JC. Hyponatremia and bone disease. Rev Endocr Metab Disord 2017;18:67-78.

32. Peri A, Giuliani C. Management of euvolemic hyponatremia attributed to SIADH in the hospital setting. Minerva Endocrinol 2014;39:33-41.

33. Wang S, Zimmermann S, Parikh K, et al. Current Diagnosis and Management of Small-Cell Lung Cancer. Mayo Clin Proc 2019;94:1599-622.

34. Kalemkerian GP. Small Cell Lung Cancer. Semin Respir Crit Care Med 2016;37:783-96.

35. Rinaldi S, Santoni M, Leoni G, et al. The prognostic and predictive role of hyponatremia in patients with advanced non-small cell lung cancer (NSCLC) with bone metastases. Support Care Cancer 2019;27:1255-61.
36. Hermes A, Waschki B, Reck M. Hyponatremia as prognostic factor in small cell lung cancer--a retrospective single institution analysis. Respir Med 2012;106:900-4.

37. Tiseo M, Buti S, Boni L, et al. Prognostic role of hyponatremia in 564 small cell lung cancer patients treated with topotecan. Lung Cancer 2014;86:91-5.

38. Wang X, Liu M, Zhang L, et al. Syndrome of Inappropriate Antidiuretic Hormone Secretion: A Poor Prognosis in Small-cell Lung Cancer. Arch Med Res 2016;47:19-24.

39. Yang Y, Sun N, Sun P, et al. Clinical Characteristics and Prognosis of Elderly Small Cell Lung Cancer Patients Complicated with Hyponatremia: A Retrospective Analysis. Anticancer Res 2017;37:4681-6.

40. Hong X, Xu Q, Yang Z, et al. The value of prognostic factors in Chinese patients with small cell lung cancer: A retrospective study of 999 patients. Clin Respir J 2018;12:433-47.

41. Fiordoliva I, Meletani T, Baleani MG, et al. Managing hyponatremia in lung cancer: latest evidence and clinical implications. Ther Adv Med Oncol 2017;9:711-9.

42. Abu Zeinah GF, Al-Kindi SG, Hassan AA, et al. Hyponatraemia in cancer: association with type of cancer and mortality. Eur J Cancer Care (Engl) 2015;24:224-31.

43. Hansen O, Sørensen P, Hansen KH. The occurrence of hyponatremia in SCLC and the influence on prognosis: a retrospective study of 453 patients treated in a single institution in a 10-year period. Lung Cancer 2010;68:111-4.

44. Li W, Chen X, Wang L, et al. The prognostic effects of hyponatremia and hyperchloremia on postoperative NSCLC patients. Curr Probl Cancer 2019;43:402-10.

45. Castillo JJ, Glezerman IG, Boklage SH, et al. The occurrence of hyponatremia and its importance as a prognostic factor in a cross-section of cancer patients. BMC Cancer 2016;16:564.

46. Filippatos TD, Makri A, Elisaf MS, et al. Hyponatremia in the elderly: challenges and solutions. Clin Interv Aging 2017;12:1957-65.

47. Chewcharat A, Thongprayoon C, Cheungpasitporn W, et al. Trajectories of Serum Sodium on In-Hospital and 1-Year Survival among Hospitalized Patients. Clin J Am Soc Nephrol 2020;15:600-7.

48. Peri A, Grohe C, Berardi R, et al. SIADH: differential diagnosis and clinical management. Endocrine 2017;55:311-9.

49. Krisanapan P, Vongsanim S, Pin-On P, et al. Efficacy of Furosemide, Oral Sodium Chloride, and Fluid Restriction for Treatment of Syndrome of Inappropriate Antidiuresis 
(SIAD): An Open-label Randomized Controlled Study (The EFFUSE-FLUID Trial). Am J Kidney Dis 2020;76:203-12.

50. Farago AF, Keane FK. Current standards for clinical management of small cell lung cancer. Transl Lung Cancer Res 2018;7:69-79.

51. Chute JP, Taylor E, Williams J, et al. A metabolic study of patients with lung cancer and hyponatremia of malignancy. Clin Cancer Res 2006;12:888-96.

52. Efthymiou C, Spyratos D, Kontakiotis T. Endocrine paraneoplastic syndromes in lung cancer. Hormones (Athens) 2018;17:351-8.

53. Filippatos TD, Liamis G, Christopoulou F, et al. Ten common pitfalls in the evaluation of patients with hyponatremia. Eur J Intern Med 2016;29:22-5.

54. Decaux G, Gankam Kengne F. Hypertonic saline, isotonic saline, water restriction, long loops diuretics, urea or vaptans to treat hyponatremia. Expert Rev Endocrinol Metab 2020;15:195-214.

55. Kai K, Tominaga N, Koitabashi K, et al. Tolvaptan corrects hyponatremia and relieves the burden of fluid/ dietary restriction and hospitalization in hyponatremic patients with terminal lung cancer: a report of two cases. CEN Case Rep 2019;8:112-8.

56. Bordi P, Tiseo M, Buti S, et al. Efficacy and safety of longterm tolvaptan treatment in a patient with SCLC and SIADH. Tumori 2015;101:e51-3.

57. Soiza RL, Cumming K, Clarke JM, et al. Hyponatremia: Special Considerations in Older Patients. J Clin Med 2014;3:944-58.

58. Li B, Fang D, Qian C, et al. The Efficacy and Safety of Tolvaptan in Patients with Hyponatremia: A Meta-Analysis of Randomized Controlled Trials. Clin Drug Investig 2017;37:327-42.

59. Castillo JJ, Vincent M, Justice E. Diagnosis and management of hyponatremia in cancer patients. Oncologist 2012;17:756-65.

60. Nervo A, D'Angelo V, Rosso D, et al. Urea in cancer patients with chronic SIAD-induced hyponatremia: Old drug, new evidence. Clin Endocrinol (Oxf) 2019;90:842-8.

61. Jaal J, Jogi T, Altraja A. Small Cell Lung Cancer Patient with Profound Hyponatremia and Acute Neurological Symptoms: An Effective Treatment with Fludrocortisone. Case Rep Oncol Med 2015;2015:286029.

62. Nagler EV, Vanmassenhove J, van der Veer SN, et al. Diagnosis and treatment of hyponatremia: a systematic review of clinical practice guidelines and consensus statements. BMC Med 2014;12:1.
63. Gralla RJ, Ahmad F, Blais JD, et al. Tolvaptan use in cancer patients with hyponatremia due to the syndrome of inappropriate antidiuretic hormone: a post hoc analysis of the SALT-1 and SALT-2 trials. Cancer Med 2017;6:723-9.

64. Berardi R, Antonuzzo A, Blasi L, et al. Practical issues for the management of hyponatremia in oncology. Endocrine 2018;61:158-64.

65. Okamoto K, Okamoto I, Takeda M, et al. A phase I study of split-dose cisplatin and etoposide with concurrent accelerated hyperfractionated thoracic radiotherapy in elderly patients with limited-disease small cell lung cancer. Jpn J Clin Oncol 2014;44:743-8.

66. Zhang X, Li XY. Prevalence of hyponatremia among older inpatients in a general hospital. Eur Geriatr Med 2020.

67. Nikoomanesh K, Choi J, Arabian S. Paraneoplastic syndrome as the presentation of limited stage small cell carcinoma. BMC Pulm Med 2018;18:169.

68. Zhang C, Jia Y, Jia Y, et al. Prognostic and predictive value of plasma D-dimer levels in patients with small-cell lung cancer. Int J Clin Oncol 2018;23:1070-5.

69. Shah MH, Lorigan P, O'Brien ME, et al. Phase I study of IMGN901, a CD56-targeting antibody-drug conjugate, in patients with CD56-positive solid tumors. Invest New Drugs 2016;34:290-9.

70. Isgrò MA, Bottoni P, Scatena R. Neuron-Specific Enolase as a Biomarker: Biochemical and Clinical Aspects. Adv Exp Med Biol 2015;867:125-43.

71. Blackhall F, Frese KK, Simpson K, et al. Will liquid biopsies improve outcomes for patients with small-cell lung cancer? Lancet Oncol 2018;19:e470-81.

72. Winzeler B, Steinmetz M, Refardt J, et al. Copeptin is not useful as a marker of malignant disease in the syndrome of inappropriate antidiuresis. Endocr Connect 2020;9:20-7.

73. Holland-Bill L, Christiansen CF, Farkas DK, et al. Diagnosis of hyponatremia and increased risk of a subsequent cancer diagnosis: results from a nationwide population-based cohort study. Acta Oncol 2018;57:522-7.

74. Svaton M, Fiala O, Pesek M, et al. Predictive and prognostic significance of sodium levels in patients with NSCLC treated by erlotinib. Anticancer Res 2014;34:7461-5.

75. Ishimoto O, Sugawara S, Inoue A, et al. Weekly irinotecan combined with carboplatin for patients with small-cell lung cancer: A phase I study. Respir Investig 2015;53:156-60.

76. Kondo R, Watanabe S, Shoji S, et al. A Phase II Study of Irinotecan for Patients with Previously Treated Small-Cell Lung Cancer. Oncology 2018;94:223-32.

77. Yang S, Zhang Z, Wang Q. Emerging therapies for small 
cell lung cancer. J Hematol Oncol 2019;12:47.

78. Saito M, Shiraishi K, Goto A, et al. Development of targeted therapy and immunotherapy for treatment of small cell lung cancer. Jpn J Clin Oncol 2018;48:603-8.

79. Tartarone A, Giordano P, Lerose R, et al. Progress and

Cite this article as: Wu R, Li C, Wang Z, Fan H, Song Y, Liu $\mathrm{H}$. A narrative review of progress in diagnosis and treatment of small cell lung cancer patients with hyponatremia. Transl Lung Cancer Res 2020;9(6):2469-2478. doi: 10.21037/tlcr-20-1147 challenges in the treatment of small cell lung cancer. Med Oncol 2017;34:110.

(English Language Editor: J. Jones) 